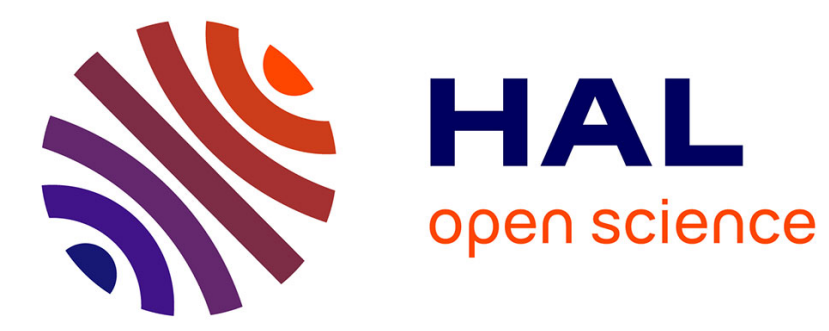

\title{
Corps en place, place du corps
}

André-Frédéric Hoyaux

\section{To cite this version:}

André-Frédéric Hoyaux. Corps en place, place du corps. L'Information géographique, 2016, 80 (2). halshs-01335193

\section{HAL Id: halshs-01335193 https://shs.hal.science/halshs-01335193}

Submitted on 21 Jun 2016

HAL is a multi-disciplinary open access archive for the deposit and dissemination of scientific research documents, whether they are published or not. The documents may come from teaching and research institutions in France or abroad, or from public or private research centers.
L'archive ouverte pluridisciplinaire HAL, est destinée au dépôt et à la diffusion de documents scientifiques de niveau recherche, publiés ou non, émanant des établissements d'enseignement et de recherche français ou étrangers, des laboratoires publics ou privés. 


\author{
A paraître en Juin 2016 dans \\ L'Information Géographique, n²016/2, 14p.
}

Hoyaux André-Frédéric

\title{
Corps en place, place du corps
}

Résumé : Cet article tente d'analyser les relations des corps, principalement humains, dans l'espace. II montre que les effets corporels ne sont pas toujours liés à un contexte qui serait par tous objectivable et quantifiable de la même façon. En effet, la relation du corps se fait plutôt à partir d'une situation que l'habitant constitue en propre. Cette relation du corps avec l'espace est une mise en chair. Elle projette l'habitant par-delà les limites supposées du contexte, de l'environnement, à travers sa pensée ou parfois grâce à la technique qui vient à son secours et crée un artefact de cette possibilité de se projeter. Cette mise en chair permet de dépasser les déterminismes qui voudraient que le corps soit toujours influencé par l'extérieur (le contexte) ou par l'intérieur immédiat (l'organisme), mais aussi par les emplacements où il se trouve et qui le désigneraient dans sa position sociale. Car si ces déterminismes semblent pouvoir lui offrir une stabilité dans le jeu des places, sa liberté ne peut se révéler qu'en déplaçant les normes qui en fondent les règles et les regards de celles et ceux qui vivent avec lui. Ainsi, au-delà du corps en place ou placé, que celui-ci bouge ou non, cet article postule que l'habitant est en quête d'un dépassement de ces canevas prédéfinis et retranscrits quotidiennement dans son existence, pour renouer avec sa propre possibilité de prendre place à travers son corps dans des espaces autres qu'il met à proximité pour pouvoir s'abstraire de la réalité présente.

Mots-Clés : corps, place, contexte, situation, cospatialité

Summary: This article investigates the relations of human bodies within space. It shows that bodies' effects are not always linked to a context equally objectifiable and measured by everybody. The relation of body with space has something to do with the situation constituted by the dweller on his own. It has something to do with the body condition as flesh or "being flesh". This relation projects the dweller beyond the supposed limits of the context and the environment. The dweller is projected through his thinking and is sometimes assisted by technical solutions that artificially enable his projection. "Being flesh" allows the dweller to overpass the body determinations that are always seen to come from the outside (the context), the immediate inside (the organism) or from the different places where he stands, giving him his social position. If body determinations offer the dweller a relative stability in the game of places, his freedom can only be revealed by displacing the norms underlying it and by displacing the way his relatives look at him. Beyond the place of the body and beyond the body in place, whatever the movement of the body, this article argues that the dweller tends to overrun the pre-established everyday frameworks that shape his existence. His objective is to revive his own possibility to take place through his body in the outer/other spaces he draws close to him to escape the current reality.

Key words: body, place, context, situation, co-spatiality

La démarche proposée ici fait suite à la réflexion d'Anne Duhamel dans le précédent numéro thématique de L'information géographique sur le corps (2014). Elle prolonge les travaux de Michel Lussault sur la question de la lutte des places, et plus particulièrement celle qui réfère au corps (2009, 100-109). Elle propose une réflexion sur les relations que les habitants entretiennent, à partir du corps, avec l'espace ou avec les autres à travers lui, en s'appuyant sur les apports de la phénoménologie et de l'interactionnisme symbolique. Ces relations sont appréhendées du point de vue des pratiques mais aussi des valeurs que les habitants leur donnent, notamment à travers la lecture symbolique des emplacements et des mouvements des corps au sein d'un espace potentiellement partagé. Ces relations sont alors l'enjeu d'une mise en place de soi et de l'autre au 
sein du monde que chaque habitant constitue en situation. Cette mise en place joue et se joue des distances et de leurs mises en mesure à travers un ensemble de " métrise " (Lévy J., 1999). Cette maîtrise des modes de mesure de la distance s'effectue à travers les valeurs que nous avons incorporées mais aussi à partir du corps considéré alors tout à la fois comme opérateur spatial (au sens où il est tout à la fois cause et effet de pratiques spatiales), créateur d'espace parce qu'il s'ouvre au monde et ouvre des mondes (Fournand A., 2005) mais aussi embrayeur spatial (parce qu'il génère des récits sur ces spatialités). L'analyse de cette mise en place est traitée ici à partir de différentes observations ${ }^{1}$ de ces corps en interaction. II s'agit de montrer en quoi cette interaction ne peut être comprise qu'en situation et non au sein d'un contexte qui serait objectivable, quantifiable, partageable a priori. Pour appréhender cela, il nous faut donc expliciter, dans un premier temps, ce que recouvre ce concept de situation, d'en dépasser la lecture sociologique; pour, dans un second temps, pouvoir alimenter la réflexion à partir de l'analyse de dispositifs situationnels types observés. Cette réinterprétation des dispositifs situationnels permet alors de réinterroger les interactions des corps. En effet, ces corps ne peuvent plus être conçus ou considérés comme inscrits uniquement dans un contexte, mais aussi comme devant être ou ne pas être à leur place selon des valeurs prétendument partagées d'un collectif social ou culturel supposé. Au contraire, comprises comme insérées dans des mondes pluriels, labiles et mobiles, mondes qui ne sont pas forcément visibles les uns pour les autres, les opérations des corps troublent ainsi l'ordre des places de soi et des autres, ce qui leur permet aussi de se libérer de cet ordre. Car si l'éducation est un apprentissage voire un " dressage " des corps et de leurs pratiques dans l'espace, c'est par la mise en lumière de cette possibilité de mondes différenciés que l'on peut entrevoir des formes de liberté.

\section{Un corps en situation}

Par le concept de situation, cher à la microsociologie (Joseph I. ; 2003 ; Quéré L., 1997), il faut comprendre que l'habitant ne pense et n'agit pas dans un contexte partagé qui serait par tous et de la même manière intellectuellement et corporellement discriminable (dans les relations) et discrétisable (à travers les classements). L'habitant constitue des situations qu'il configure selon différentes mises à proximité et mises à distance des éléments de la réalité, mais aussi selon différents critères et différents référents qui lui permettent de légitimer son action avant même parfois qu'elle ne se réalise réellement. En ce sens, l'appréhension du monde, d'une situation qu'opère l'habitant à travers son corps ne relève pas d'une mise en relation objective des conditions de la réalité présente. Dans l'idée de situation, de ce monde constitué, on retrouve l'idée d'espace corporel énoncé par Merleau-Ponty. Un espace qui « n'est plus celui de la Dioptrique (de Descartes), réseau de relations entre objets, tel que le verrait un tiers, témoin de ma vision, ou un géomètre qui la reconstruit ou la survole, [mais] un espace compté à partir de moi comme point ou degré zéro de la spatialité. Je ne le vois pas selon son enveloppe extérieure, je le vis du dedans, j'y suis englobé.

\footnotetext{
${ }^{1}$ L'objectif n'étant pas ici purement méthodologique, je me contenterai de dire que cette observation suit certains attendus de la microsociologie qui est « avare en généralisations : par induction analytique, elle peut remonter vers des propositions à validité trans-situationnelle, mais elle ne le fait qu'avec infiniment de prudence, et sans jamais sous-estimer la contextualité de son activité de généralisation » (Céfaï D., 2007, 14). Cette généralisation peut se faire au regard de la propre interprétation des descriptions en situation qui est opérée par le chercheur. En ce sens, il est son propre informateur et son propre régulateur. II n'est pas dans une opération de classement, pour capter les récurrences des faires et des dires mais bien plutôt pour appréhender comment se fondent les situations elles-mêmes à travers ces faires et ces dires situés. "Cette position porte à privilégier avant tout l'observation de situations empiriques d'interaction et de co-construction d'un monde : puisque les activités (non seulement linguistiques et discursives) sont constitutivement liées à leur contexte, qu'elles contribuent à élaborer en s'organisant " (Mondada L., 2001, 199). En cela, ce travail s'apparente à la mise en œuvre d'une " observation flottante " au sens de Colette Pétonnet. "Elle consiste à rester en toute circonstance vacant et disponible, à ne pas mobiliser l'attention sur un objet précis, mais à la laisser "flotter" afin que les informations la pénètrent sans filtre, sans a priori, jusqu'à ce que des points de repères, des convergences, apparaissent et que l'on parvienne alors à découvrir des règles sous-jacentes » $(1982,39)$.
} 
Après tout, le monde est autour de moi, non devant moi " (Merleau-Ponty, 1985, pp. 58-59). Un point zéro à partir duquel l'habitant mesure et prend la mesure de son monde et des autres habitants qui l'habitent. S'il spatialise la réalité autour de lui, il lui donne également sens en la spatialisant. Ce sens nourrit ses choix de ce/ceux qu'il vise comme participant de son monde.

Cette mise en situation est donc une rencontre entre un contexte et un habitant qui l'appréhende et se l'approprie tout en l'appréhendant. Cet habitant peut pourtant penser à bon droit partager la perception même des éléments humains (autres acteurs) ou non humains (les actants au sens d'objets qui nous font réaliser des opérations spatiales) qui se trouvent dans ce contexte pensant que les limites mêmes de sa perception, l'horizon de sa portée sont identiques. Mais, bien qu'il permette la communication et donc les relations sociales, ce partage est fictif. II est donc une traduction d'une situation, une traduction partagée qui rebat en fin de compte les cartes de nos prétendus déterminismes. Car en posant le paradoxe suivant: " ou bien je ne réfléchis pas, je vis dans les choses et je considère vaguement l'espace tantôt comme le milieu des choses, tantôt comme leur attribut commun, - ou bien je réfléchis, je ressaisis l'espace à sa source, je pense actuellement les relations qui sont sous ce mot et je m'aperçois alors qu'elles ne vivent que par un sujet qui les décrive et qui les porte, je passe de l'espace spatialisé à l'espace spatialisant " (MerleauPonty M., 1945, 282) ; Merleau-Ponty nous libère des déterminismes spatiaux mais nous assigne à nous entendre sur ce qui ferait le commun entre nous. II nous assigne à institutionnaliser nos propres déterminismes sociaux à travers un partage des regards sur le monde, et donc des jeux de placements des corps qui se devraient d'être à certains endroits plutôt que d'autres.

Isaac Joseph définit le contexte comme un "cadre local et perceptif dans lequel se déroule une activité et un espace de parole auquel les participants se réfèrent au cours de l'échange. Du point de vue d'une écologie des activités, le terme désigne l'environnement et les ressources disponibles. Du point de vue de la cognition située, il renvoie aux indices permettant aux participants de faire des inférences sur l'action ou la conversation en cours " (Joseph I., 2003, 123). L'importance de la lecture de ce contexte requiert donc une référentialité de l'habitant aux éléments configurateurs, à la fois aux acteurs et aux actants de ce contexte, c'est-à-dire aux éléments qui lui font faire une action et plus particulièrement spatiale soit à travers un mouvement ou l'évocation d'un mouvement. Pour autant, ni les limites de ce cadre local ni les éléments qui le composent ne sont totalement perçus de la même manière par chaque intervenant d'une situation. Et les intervenants eux-mêmes, en ce qui concerne les acteurs, ne peuvent être conçus comme des êtres figés dans leur manière d'être et de penser. Ils évoluent, changent justement en fonction de la situation et en fonction de ce qu'ils mettent en situation, c'est-à-dire l'éclairage, la mise en visibilité de ce qu'ils font, sont, ont. C'est aussi par ses mises en visibilité qu'il opère dans l'espace à travers ces dires et ces faires que I'habitant pense proposer son partage des regards. II évoque par ses pratiques corporelles, l'extimisation de ses émotions, son habillement, un ensemble de signes qui renvoient à des régimes de visibilité (Lussault in Lévy et Lussault, 2003, 997) qu'il pense ou espère partager et qui réfère avec ce qu'il faut faire ou ne pas faire à certain emplacement spatial et en fonction du collectif social avec qui l'on se trouve : montrer son corps nu, pleurer ou ne pas pleurer, avoir un couvre-chef, etc.

Cette situation, à la différence de la microsociologie n'est pas comprise ici comme confinée à la coprésence des membres et des éléments du contexte. La situation sociale correspondrait selon Isaac Joseph à un "espace-temps défini conventionnellement où deux personnes ou plus sont coprésentes ou communiquent et contrôlent mutuellement leurs apparences, leur langage corporel et leurs activités " (Joseph I., 2003, 124). Cette coprésence invite aujourd'hui à d'autres perspectives que le seul côtoiement. Une situation naît aussi de relations qui dépassent la sphère de l'environnement, du contexte immédiat. Ce dépassement peut être induit par l'utilisation d'outils techniques permettant cette mise à proximité, comme avec le téléphone portable (Jauréguiberry F., 2003) ou tout outil de télécommunication. Mais cette mise à proximité se révèle tout autant par l'outil indispensable de notre humanité, l'esprit, l'imagination ou la pensée (sans présumer ici la portée épistémologique des termes usités). Car une situation d'interaction sociale sur " site naturel " comme aiment à utiliser les microsociologues ne recourt pas uniquement aux éléments présents mais aussi à un ensemble d'éléments présentifiés, c'est-à-dire mis à jour dans 
l'esprit de l'habitant voire mise en visibilité par la pensée de celui-ci à son interlocuteur pour constituer la situation et donc l'ensemble des jeux de spatialités qui s'y développent.

Ainsi, cette situation ne relève pas uniquement des éléments présents, qu'ils soient acteurs ou actants du contexte mais aussi des éléments qui en sont absents, soit parce qu'ils en sont éloignés " physiquement " (mon partenaire qui se trouve à l'autre bout du monde), soit parce qu'ils ne sont plus visibles mais ont pu exister à cet emplacement dans un passé plus ou moins lointain (le lieu de décès de son ami, sa maison d'enfance qui a été détruite). La situation est donc une combinatoire d'éléments présents et absents qui sont mis à proximité par l'habitant, qui en existant, les ramène à lui, donc en élude la distance, à travers les mouvements du corps et de la pensée. A l'inverse, des éléments (acteurs ou actants) objectivement présents peuvent être mis à distance ou purement et simplement inexistant au regard de la mise en situation de l'habitant. Les jeux de spatialités (micro au niveau des émotions, des kinesthèses ${ }^{2}$, ou macro à travers les mobilités) qu'engagent ces corps situés et socialisés, placés, ne relèvent donc pas seulement des contextes dans lesquels ils se trouvent mais aussi (et de plus en plus ?) des espaces au sein desquels ils se projettent en pensées auprès de ces éléments visibles ou invisibles mais présentifiés. La présentification est comprise ici comme " la représentation intuitive de quelque chose dans son absence, d'un quelque chose absent. Elle est une représentation dans l'intuition d'un objet en son absence " (Houillon V., 1996, 220). Les actes de la présentification réfèrent à " ceux de la remémoration, de la représentation imaginaire ou de la représentation par l'image au sens habituel de ce mot " (Husserl E., 1974, 281). Sentir l'odeur d'un parfum qui nous projette auprès d'un être cher comme s'il était réellement à côté de nous et retrouver l'émotion et la sensation de sa présence. Et engager peut-être des actions futures pour le revoir. Cette présentification liée à l'odeur relève d'une opération spatiale (celle de la mise à proximité de cet être cher) comme elle engage potentiellement une nouvelle opération spatiale (celle de se rapprocher physiquement et plus seulement mentalement de cet être cher) Le corporel, au sens du mouvement sensible immédiat des corps, se trouve donc couplé au corporéique, au sens du mouvement sensible de ses pensées. Egalement sensible car l'éloigné en pensée peut occasionner des sensations, des émotions (contraction des muscles, chair de poule, pleurs, rires) au même titre que ce qui semble proche, coprésent. Cet acte sensible étant lui-même signe d'une façon d'être qui rejaillit sur le jeu de placement des autres membres de la situation.

Le corps placé renvoie donc à l'idée que ce placement se joue à la fois comme un moyen d'occuper (auto-assignation) ou de faire occuper (assignation) un emplacement pour tenir une position (autodésignation) ou rappeler la position à tenir (désignation) pour soi-même ou pour un ou des acteurs dans la société. Si l'idée d'emplacement traite de l'occupation par le corps situé d'un espace (topos) il ne doit pas s'abstraire d'un côté de sa corporalité (à travers la gestuelle corporelle); de l'autre de sa corporéité, sa capacité à se projeter dans un ailleurs par sa conscience ou tout autre moyen technologique (écouteurs, téléphones portables, internet, etc.). Car le corps s'il peut être visible à un endroit pour les autres peut être absent de l'espace de cette présence pour celui qui le fait bouger. II est projeté sur un espace autre qui délimite alors tout autant le jeu des corps et des places dans l'univers présent et dans l'univers présentifié (exemple des individus téléphonant ou écoutant de la musique et ne regardant pas le monde dans lequel ils transitent, déconnectés qu'ils sont avec l'univers présent et connectés qu'ils sont avec l'univers mis en lien, mis à proximité bien qu'éloigné objectivement). Mais le corps peut aussi, de par son enveloppe (des vêtements par exemple), exprimer sans le vouloir, ou mettre en visibilité des significations qui ne correspondent pas avec l'endroit où il est censé être (une robe de soirée pour un colloque, un short au sein d'une église) pour celles et ceux qui le regardent. La désignation de ce corps à travers la typification renvoie alors à un travail sur l'emplacement adéquat pour trouver ou avoir une place légitime dans l'espace. II y a bien une police des places (Lussault M., 2009, 110-119) plus ou moins partagées, plus ou moins contractualisés par les membres des collectifs institués ou auto-institués en fonction des situations

\footnotetext{
${ }^{2}$ Les kinesthèses sont liées à des stimulations sensorielles. Elles peuvent être conçues de deux manières différentes, soit de manière physiologique en tant que réaction musculaire ou articulaire, soit de manière phénoménologique en tant qu'orientation du corps en mouvement, à l'expérience du corps qui se meut.
} 
que les acteurs observent et constituent. Les corps (le nôtre et celui des autres) sont donc des embrayeurs de discours et de pratiques. Ce sont des opérateurs spatiaux qui motivent consciemment ou inconsciemment nos perceptions, nos sensations, donc nos constitutions de la situation mais aussi nos mouvements, nos trajets, nos spatialités pour en conserver ou en modifier la réalité (pour changer la situation ou la faire perdurer). Et potentiellement les perceptions, sensations, constitutions des autres acteurs coprésents ou non.

$S^{\prime}$ il y a police, il y a donc normes, codes et contraintes. Mais c'est justement par sa capacité de déréaliser le coprésent du contexte et de le reconfigurer en situation à travers des éléments présents mais aussi non présents que l'habitant se libère de ce totalitarisme du visible partagé. Car comme une caméra de vidéosurveillance, nos yeux pensent pouvoir voir la réalité coprésente mais ils ne peuvent percevoir ce qui est configuré dans la tête des autres acteurs qui participent potentiellement de cette réalité. En cela, nos corps peuvent se déplacer. Mais cette liberté pose potentiellement un problème dans les relations corporelles. En effet, si nous ne partageons pas systématiquement la même réalité fondée uniquement sur les éléments coprésents, il faut alors concevoir un choc des situations qui ouvre la porte à des événements spatiaux inattendus, non prévus. Les habitants vivent alors dans des mondes parallèles constitués à chaque instant à partir des éléments du contexte et de ceux que leur conscience les amène à présentifier dans un jeu de cospatialité. En effet, comme d'autres commutateurs, la conscience de l'habitant lui offre "la capacité de jouir en même temps de plusieurs espaces de taille différente " (Lussault M., 2007, 63), mais aussi d'en ressentir les effets immédiats même si ces espaces sont à distance.

\section{Dé-placer les corps, une conquête de l'humanité}

L'être humain, dès son plus jeune âge apprend à reconnaître à travers un ensemble de situations comment placer son corps et celui des autres au sein de l'espace. Cela lui permet ainsi de se sécuriser à travers un ensemble d'anticipation dans la relation qu'il doit tenir face aux autres. Erving Goffman énonce l'idée que "la société établit des procédés servant à répartir en catégories les personnes et les contingents d'attributs qu'elle estime ordinaires et naturels chez les membres de chacune de ses catégories. Les cadres sociaux établissent les catégories de personnes qu'il est probable d'y rencontrer. La routine des rapports sociaux dans les cadres établis nous permet d'avoir affaire aux autres, habituellement présents, sans leur accorder une attention ou des pensées particulières. Par suite, lorsqu'un inconnu se présente à nous, ses premières apparitions ont toutes chances de nous mettre en mesure de prévoir la catégorie à laquelle il appartient et les attributs qu'il possède, son "identité sociale", pour employer un terme meilleur que celui de "statut social", car il s'y inclue des attributs personnels tels que l' "honnêteté", tout autant que des attributs structuraux comme la "profession". Nous appuyant alors sur ces anticipations, nous les transformons en attentes normatives, en exigences présentées à bon droit " (Goffman E., 1975, 11-12). L'extimisation (Tisseron S., 2001 ; 2011 ; Lussault M. in Lévy J. et Lussault M., 2003, 335) du corps placé des autres êtres humains permet donc à chacun d'entre nous d'anticiper nos propres manières d'êtres et de faire, notamment à travers des jeux de mise à proximité et mise à distance des autres acteurs mais aussi des actants, et cela sans que le langage n'intervienne comme avertisseur potentiel.

Pour Sloterdijk, nous serions dès le plus jeune âge conditionné à cette mise en sécurité, à travers les mouvements des corps les uns par rapport aux autres, au sein de ce qu'il appelle un parc autogène. " Avant le langage, il existe des gestes de prise de distance à l'égard du monde, des gestes du type dur (relevant de la technique du coup, du jet et de la découpe) qui produisent et sécurisent la couveuse humaine. Le lieu spécifique de l'être humain possède les qualités d'un utérus externe aménagé par la technique, dans lequel les êtres nés continuent à jouir des privilèges réservés à ceux qui ne le sont pas. Dès lors, les hommes vivent toujours, et presque exclusivement, dans une situation épargnée pour laquelle on peut utiliser l'expression de parc autogène " (Sloterdijk P., 2000, 55). Mais pour suivre l'idée de Sloterdijk, il faut insister sur le fait que ce parc autogène ne doit pas être conçu comme un environnement clos au sein duquel nos manières de faire, de penser, de dire sont conditionnées socialement et spatialement. Au contraire, ce parc autogène est labile, et s'il exprime une volonté de sécurité ontologique au sein d'un ensemble de routines, il relève aussi d'un 
ensemble de situations, d'un monde constitué par chaque habitant à travers les mouvements de son corps (sa corporalité) et surtout la pensée qu'il développe à partir ou à travers ce corps (sa corporéité) pour aller par-delà cet attendu et construire son humanité.

Pour Giddens, « à des degrés qui varient selon les contextes et les caprices de la personnalité de chacun, la vie quotidienne suppose une sécurité ontologique qui est l'expression d'une autonomie de contrôle corporel dans des routines prévisibles » $(1987,99)$. La sécurité ontologique se structure ainsi sur la routine des actes quotidiens, l'amoncellement progressif des expériences banales de l'individu. Cette sécurité permet alors à ce dernier d'affronter la multitude des défis qui s'imposent à lui dans le monde qui l'entoure sans pour autant que son équilibre intérieur et ses motivations en soient trop affectés (Giddens A., 1987, 443). Si cette sécurité est bafouée, alors, il affronte une situation critique qui ébranle sa place et sa compétence à l'arrangement général, sociétal des places (Lussault M., 2009, 84-85). Par "situation critique ", Giddens fait "référence à des événements radicalement perturbants et de nature imprévisible qui menacent ou détruisent la "certitude" des routines institutionnalisées chez un grand nombre d'individus " (Giddens A., 1987, 110). C'est le cas, lorsqu'il y a pour l'habitant, impossibilité de conserver les mêmes "modes de conduite inspirés de leurs modes de vie antérieurs" et "imprévisibilité générale des événements " (routines brisées); impossibilité de conserver des relations avec les mêmes personnes et perte "du sens du monde extérieur " (Giddens A., 1987, 109-113). Mais aller au-delà de ces routines, par l'utilisation consciente ou inconsciente de sa corporéité, de cette faculté de présentification, c'est aussi percer l'environnement, sa cage ontologique, c'est permettre un déplacement de soi, à travers son corps.

Cette humanité est présentée ici comme se constituant à travers la capacité qu'a l'habitant de se projeter par-delà ses ancrages, ses identités, d'être capable de se dé-placer, c'est-à-dire de s'obliger à modifier ses regards sur ce que sont les autres et lui-même à travers des supposés emplacements et statuts. En effet, s'il devait en rester à la mécanisation de ses interactions selon une échelle de domination définitivement déterminée et où les places supposées de chacun au sein de son environnement, seraient inamovibles, il pourrait être conçu comme enfermé dans son animalité : «Ce qui est animal vit toujours dans cette cage ontologique que les modernes [...] nomment l'environnement (comme "monde autour"), tandis que la spécificité de l'être humain est d'accomplir la sortie de l'environnement, la percée dans l'absence de cage ontologique, pour laquelle nous ne trouverons sans doute jamais meilleure caractérisation que le mot le plus trivial et le plus profond des langages humains, l'expression de "monde" ॥ (Sloterdik P., 2000, 27). L'être humain serait donc capable de dépasser cette mécanisation qui relèverait d'un ensemble de normes implicites incorporées qui révèleraient les violences symboliques faites aux habitants selon leur place dans la société (Bourdieu P., [1982]2001). Habitants qui reproduiraient en quelque sorte, sans en être conscients, à travers leur corps, une relation instinctive, allant de soi, à leur environnement sociospatial. Cette relation les priverait d'une forme de liberté par rapport à ces convenances, et pire ces dominations établies à travers la façon de penser les choses ${ }^{3}$. Pour préserver cette part d'humanité, l'être humain aurait la faculté de dépasser ces cadres et de réaliser ce que Sloterdijk appelle la percée de l'environnement. Cette percée permet d'aller au-delà de ce qui est attendu tant dans

\footnotetext{
${ }^{3}$ Au sens strict, la violence symbolique recourt à l'idée d'imposer un sens à travers des manières d'être, de faire et de penser, mais un sens qui reflète et révèle surtout des normes de comportements qui seraient incorporées et liées à un positionnement social des places qui serait immuable, un habitus. Ces normes comportementales, traduites par des façons de se tenir, de marcher, de manger, de parler, de sentir, seraient alors autant de révélations de ce que Bourdieu appelle l'hexis corporelle. L'idée avancée ici est que l'habitant peut dépasser le sens incorporé de cette violence symbolique. Car quels que soient les classements effectués implicitement par toute institution pourvoyeuse de cette violence (l'école, l'église, la famille, etc.), l'habitant peut à tout moment en contourner, en détourner, en dépasser les fondements. Car il est capable de se mettre en situation différemment de ce qui est apparemment attendu par ceux qui en délimitent les façons d'être, de faire et de penser. L'habitant est ainsi en mesure, par des jeux de mises à proximité et de mises à distance à travers le corps, de déréaliser le présent prévisible et de se projeter par-delà les cadres prescrits, en acte et en sens.
} 
notre relation sociale aux autres et à nous-mêmes que dans nos relations à l'espace. Ainsi, l'habitant pourrait se projeter en dehors de l'environnement, du contexte pour constituer sa propre situation.

En ce sens, la conscience de l'habitant fait lieu à chaque instant de la constitution de sa réalité. Mais il ne fait pas lieu à cause de sa coprésence aux êtres et aux choses qui l'entourent, ou en tout cas pas seulement, mais à partir de sa capacité d'être toujours déjà auprès de ces êtres et de ces choses, de ses êtres et de ses choses. L'habitant, en constituant une relation spatiale, sociale ou temporelle, constitue un sans distance, même dans la distance (au sens objectif du passé ou du lointain), et cela grâce à sa conscience. Même en étant au loin de quelqu'un ou de quelque chose, l'habitant peut à travers sa pensée se projeter, donc être jeté auprès de ce quelqu'un ou ce quelque chose et donc de déréaliser mentalement cette distance par la cospatialité, la coexistence pour reprendre les termes de Merleau-Ponty. Penser mettre au loin ou auprès quelqu'un ou quelque chose, c'est faire lieu de cette mise au loin ou auprès, c'est actualiser une situation et c'est donner sens pour l'autre et pour soi à cette actualisation. "De même que l'on ne peut comprendre la mémoire que comme une possession directe du passé sans contenus interposés, on ne peut comprendre la perception de la distance que comme un être au lointain qui le rejoint là où il apparaît " (Merleau-Ponty M., 1945, 307).

Cette conscience est aussi conscience d'un corps au sein de l'espace. L'habitant constitue le monde à travers l'incorporation qu'il met en place de sa réalité. Pour Merleau-Ponty, notre corps est le point origine (Nullpunkt) de toutes nos appréhensions, nos ouvertures au monde, c'est lui qui métrise (en tant que maîtrise des métriques) la situation. Cette métrise permet de donner chair au monde, à notre monde, par notre projection corporelle située auprès de lui. " Mon corps est fait de la même chair que le monde (c'est un perçu), et [...] cette chair de mon corps est participée par le monde, il la reflète, il empiète sur elle et elle empiète sur lui (le senti à la fois comble de subjectivité et comble de matérialité), ils sont dans un rapport de transgression ou d'enjambement. [...] mon corps n'est pas seulement un perçu parmi les perçus, il est mesurant de tous, Nullpunkt de toutes les dimensions du monde. II n'est pas un mobile ou mouvant parmi tous les mobiles ou mouvants, je n'ai pas conscience de son mouvement comme éloignement par rapport à moi, il sich bewegt ${ }^{4}$ alors que les choses sont mues " (Merleau-Ponty M., 1964, 302).

Cette projection située n'est pas douée de réflexivité sur son propre mouvement du corps mais bien plutôt sur le mouvement parmi les choses sur lesquels ce corps se projette. "Le se toucher, se voir du corps est à comprendre lui-même d'après ce que nous avons dit du voir et du visible, du toucher et du touchable, ce n'est pas un acte, c'est un être à. Se toucher, se voir, d'après cela, ce n'est pas se saisir comme ob-jet, c'est être ouvert à soi, destiné à soi. Ce n'est pas davantage, donc, s'atteindre, c'est au contraire s'échapper, s'ignorer, le soi en question est d'écart, [...], qui donc ne cesse pas d'être caché ou latent » (Merleau-Ponty M., 1964, 302-303). Donc cette projection située ne doit pas seulement être comprise en tant qu'orientation de notre vision qui embrasse un paysage qui se trouverait face à nous mais être comprise aussi par l'orientation d'une pensée qui se projette pardelà ce paysage sans forcément appréhender réflexivement qu'elle $s^{\prime} y$ trouve. Regarder au loin la mer tout en pensant à ce que nous devons réaliser la semaine prochaine en rentrant des vacances ne nous amène pas à réfléchir sur le pourquoi nous pensons à ce que nous devons faire la semaine prochaine, nous y sommes plongés de fait. Cela nous préoccupe. Et les « impressions » que pourrait

\footnotetext{
${ }^{4}$ Cette expression allemande utilisée comme tel et sans traduction par Merleau-Ponty peut s'expliciter par « il se déplace ". Si l'expression renvoie à l'idée de "se déplacer", dans un cadre géographique, on pourrait admettre que Merleau-Ponty distingue les acteurs, des actants (ou des objets). Les acteurs se déplacent quand les actants sont mus. Les premiers avec intentionnalité, les seconds, sans. Mais s'il se déplace, l'habitant ne le fait pas dans un espace objectif partagé puisque cet espace du mouvement est absent à son propre regard. En effet, il n'est jamais que l'espace vers lequel il porte son regard, que ce dernier se fixe sur le contexte ou pardelà celui-ci. " Si le soi passe dans son mouvement et s'éloigne dans le monde, il se retrouve lui-même en ce dépassement, de sorte que cet éloignement est sans distance. C'est pourquoi Merleau-Ponty remarque que mon mouvement, vécu comme tel, demeure invisible comme mouvement objectif » (Barbaras R., [1992]2008, 32).
} 
nous donner la mer n'ont donc pas de sens pour nous à ce moment-là. Un monde autre recouvre ce contexte que nous semblons pourtant percevoir de nos yeux.

L'habitant se constitue alors pleinement une situation qui le préoccupe et qui fait sens de sa réalité présente. Pour autant, cette pensée fugace de ce qu'il faut qu'il fasse la semaine prochaine peut l'amener tout d'un coup à une forme d'angoisse, de fébrilité qui peut se traduire par une transformation du corps, transformation qui est en déconnexion avec le sentiment corporel de bienêtre qu'il pouvait avoir juste avant, en étant réellement plongé dans le paysage superbe qu'il avait devant lui. Dès lors, parler de projection située, c'est accepter l'idée que l'habitant est toujours déjà ailleurs que dans les objets circonstanciels qui l'entourent, que son corps bouge ou ne bouge pas. II est mu par sa projection auprès des diverses situations sur lesquelles il se projette. Finalement, son corps n'est jamais fixe car même quand il ne bouge pas, il est toujours auprès de. Cette projection mentale se fait pourtant sans forcément qu'il est conscience d'une trajectoire qui irait d'un point à un autre, d'un ici à un ailleurs. "Tout espace pour la réflexion est porté par une pensée qui en relie les parties, mais cette pensée ne se fait de nulle part » (Merleau-Ponty M., 1945, 328).

Donc, nous sommes un point origine qui n'est jamais nulle part, nous sommes en mouvement perpétuel et même nos pensées sont toujours en mouvement perpétuel. C'est donc peut-être cela qui nous pousse à en stabiliser la réalité par des routines qui peu ou prou nous apporteront un minimum de sécurité ontologique. C'est dans cette force des habitudes que chacun engramme des éléments personnels de la constitution des situations à venir, qui sont censés advenir en fonction d'où il est, avec qui il est et comment son corps est. Sentir le regard de l'autre posé sur soi résulte tout autant de l'acte de ce regard objectivement posé sur soi que du fait que nous avons appris à sentir, voire à attendre le principe même de ce regard, que cette attente soit vécue positivement ou négativement. Encore que ce regard qui est semble-t-il posé sur lui parce que l'axe des yeux vient dans sa direction peut être un regard qui se porte en réalité sur bien d'autres choses que lui. L'habitant ne peut en effet appréhender le tangible de ce regard pour l'autre. II aura donc toujours plus de certitudes dans l'impression qu'il est vu que dans celle qu'il est regardé.

Au sens strict d'une appréhension objective de la réalité, tout n'est donc qu'illusion. Mais c'est cette illusion qui fait foi de ce que je ressens, de ce que je perçois donc c'est cela qui va fonder mon activité, celle de détourner mon propre regard, celle de mettre en accusation ce regard par quelques remarques ou par une attitude particulière. Car même l'objectivation de la perception n'est qu'illusion. L'habitant vit donc dans une illusion et le sens qu'il produit de cette perception n'est qu'une fiction. Mais illusion et fiction font la chair du monde de cet habitant, la réalité qu'il constitue (Merleau-Ponty M., 1945, 343).

On pourrait alors penser que cette manière de traduire les choses relève de la pure spéculation mais les derniers travaux en physiologie de l'action rejoignent les avancées des phénoménologues comme le montrent les recherches conjointes d'Alain Berthoz et Jean-Luc Petit. Ces chercheurs montrent que l'être anticipe toujours déjà la situation qu'il vit. En effet, sa projection ne se déroule de manière réflexive qu'à partir de l'objectivation d'une réalité au sein de laquelle il créerait une situation qui lui convienne. Sa projection est une configuration du monde au sein duquel il réalise ses actions : " avec son cerveau, l'organisme dispose de toutes les ressources nécessaires pour ce qu'il ne convient plus, dès lors, de décrire comme traitement perceptif d'une information sensorielle externe, mais qu'on décrira plutôt comme l'affection de soi par soi continuelle d'un être spontanément agissant et constamment sensible aux effets sur lui de sa propre action, à mesure (voire avant même) qu'il les éprouve dans son corps et les objective dans son environnement, corps et environnement qu'il projette à l'avance, découvre et s'approprie par la même occasion. Aux théories de l'imprégnation passive de l'organisme par une information d'origine externe devra donc succéder une théorie de la constitution du (modèle interne du) monde par l'organisme lui-même qui a ce monde (de même qu'il a aussi son corps et son esprit à s'approprier en s'en faisant un modèle interne) " (Berthoz A. \& Petit J.-L., 2006, 35-36). Sauf cas exceptionnel, l'habitant anticipe donc toujours la réalité qui lui advient. Mais s'il anticipe sa propre réalité en se jouant du contexte, il se trouve confronté aux mêmes stratégies de détournement de la part de ses coexistants. Cette confrontation peut provoquer alors un choc de situations. 


\section{Coprésence et cospatialité, le choc des situations.}

J'ai montré que l'espace est considéré ici en tant qu'il permet une assignation (de soi/des autres par soi et/ou par les autres) à travers la localisation et le mouvement de ces corps (acteurs et actants) et en quoi le sens de cette localisation et de ce mouvement traduisent une désignation sociale (de soi/des autres par soi et/ou les autres) mise en jeu à travers un ensemble d'interactions symboliques. L'habitant en tant qu'acteur individuel ou social (parce qu'il participe à un ou des collectifs institués il est membre d'une entreprise, d'une association, d'une famille - ou, auto-institués - il est fan d'un chanteur, amoureux d'une région) attribuerait a priori, selon les situations, la bonne place aux corps qui l'entourent, c'est-à-dire où ils se doivent ou se devraient d'être en fonction de qui ils sont ou croient être. L'enseignant est ainsi assigné à être derrière son pupitre assis sur sa chaire et au-delà d'être habillé d'une certaine façon et avoir un certain comportement attendu pour "faire » enseignant. Mais à l'inverse, tout individu qui se trouverait en début d'année à l'emplacement dévolu à l'enseignant, et aurait un comportement attendu du corps passerait, pour les étudiants, même s'il n'en a pas le statut, pour un enseignant. Le corps en place, déterminé par son emplacement spatial, génère donc symboliquement, pratiquement et implicitement la désignation sociale supposée du statut, du rôle et de la fonction de la personne placée. De même, dans une situation donnée, anticipativement constituée par l'habitant, toute présence d'autres acteurs ou actants non prévue dans le type de situation répertorié par ce dernier entraîne une appréhension troublée, perturbée dans sa prétendue normalité et génère ce que l'on pourrait appeler un événement spatial ${ }^{5}$. En effet, " un événement spatial concerne ce qui apparaît dans l'organisation spatiale [ici la situation] et qui n'y était pas précédemment. Cette apparition est nécessairement brusque à l'échelle temporelle du système spatial dans lequel il se produit » (EPEES, 2000, 194). Mais cet événement peut aussi jouer sur une disparition de ce qui était prévu dans cette organisation.

Un jeune enfant est à table, sa mère vient d'aller lui chercher son yaourt dans le frigo, il lui demande avec insistance du sucre. Elle place le sachet sur la table. Mais au moment même où il allait le prendre, sa cuillère tombe par terre. II va pour la ramasser en détournant la tête de la vue du sachet. Entretemps, une deuxième personne attablée prend le sachet pour sucrer son propre dessert et le repose à un autre endroit. L'enfant, de nouveau assis, sans regarder l'objet qu'il avait prévu de prendre avant que sa cuillère ne tombe, lance son bras vers le sachet de sucre et là plus rien.

Confusion. Où est le paquet, a-t-il jamais existé ? II n'est plus à l'emplacement initial que l'enfant avait anticipé qu'il soit. Le projet qu'il avait de se projeter corporellement vers le sachet, de le mettre à sa proximité, de maîtriser les distances à ce dernier s'en trouve bouleversée. Il doit réinitialiser la situation. Cette proposition invite alors avant tout à un travail d'observation des corps agissant. Mais elle ne peut faire fi d'une construction narrative de la réalité, car celle-ci permet d'entériner des manières d'être, de faire et de pratiquer l'espace, d'appréhender aussi le passage conceptuel entre le contexte objectivable et la situation vécue.

Le corps est opérateur spatial, mais il l'est alors tout autant en tant qu'acteur qu'actant. Le corps de l'autre peut être opérateur spatial de notre propre corps sans que cet autre corps n'ait conscience de ce qu'il fait pour nous. Au contraire, la corporalité de l'autre est une invitation à cette spatialité. Dans tous les cas, le placement du corps de par sa désignation symbolique conditionne notre propre placement ou provoque notre dé-placement c'est-à-dire le fait de croire que l'on a changé de position sociale soit à travers la fonction, le statut ou le rôle que l'on se donnait ou que l'on nous donnait a priori. Mais les actants ont leur propre place dans cet univers symbolique et pratique. Ils jalonnent, à travers leur matérialité, les mises en situation de la réalité et peuvent être des embrayeurs de placement des uns et des autres. En effet, un objet doit être temporairement ou durablement à un emplacement, le sachet de sucre doit être dans son tiroir ou sur un endroit de la

\footnotetext{
${ }^{5}$ Pour la suite de notre propos, nous indiquerons en italique les dispositifs situationnels types que nous avons observés et sur lequel se fondera notre analyse. Ces dispositifs recouvrent une forme d'objectivation à travers une typologie. Mais cette objectivation ne recouvre pas celles réalisées par les habitants de leur contexte.
} 
table. S'il ne l'est pas, il provoque des discours d'interrogation, de provocation, mais aussi des corporalités exprimant aussi qui se doit de faire (la mère se doit de se lever pour aller chercher le sucre).

Une mère de famille tire sa fille de 3-4 ans par la main en traversant une rue sans se préoccuper de sa difficulté à la suivre ni des voitures qui arrivent. Elle a des écouteurs sur les oreilles. A-t-elle seulement entendu. Absente à la scène.

Comme nous l'avons laissé sous-entendre précédemment, l'habitant ne peut percevoir la place de l'autre (et donc son corps qui possède un emplacement) que s'il participe de la situation qu'il constitue. Parfois, les corps s'entrechoquent involontairement parce qu'ils ne se sont pas vus mais dans cette idée du pas vu, il y a l'idée qu'ils n'ont pas été attentifs l'un à l'autre. En ce sens, le corps peut donc être présent-absent. Cette présence-absence relève du fait que parfois, les yeux auraient permis " objectivement " de voir l'autre, mais ils ne participaient pas de la situation constituée par cet autre. Parfois, l'inattention provient du fait que les autres sens qui auraient pu conforter la vue à cette présence (le sentir, l'entendre) sont volontairement obstrués par des outils techniques qui nous isolent volontairement de ce contexte et nous mettent dans l'impossibilité de concevoir la situation... contextuelle. En ce sens, le corps peut-être aussi absent-présent car même sans être présent dans le contexte objectivé, il se trouve à côté de nous en situation.

II ne peut donc y avoir de distance entre deux corps (acteurs-acteurs la mère et sa fille ; ou acteursactants : la mère et la voiture) si ces deux corps ne participent pas de la même situation au sens de leur constitution respective et non au sens d'un contexte objectivé. Ainsi, il ne peut y avoir de métrise, c'est-à-dire de maîtrise des distances qui séparent les uns des autres, que dans une situation où les protagonistes la coconstituent de manière plus ou moins partagée.

Deux personnes discutent côte à côte en effectuant un trajet sur une rue. Arrive sur eux une autre personne qui ne pourra apparemment pas passer sans se faire bousculer. Chaque personne participant de ce contexte peut voir clairement l'autre. Pour autant, les deux personnes ne modifient pas leur trajectoire et le choc épaule contre épaule intervient.

Pris dans une relation d'interaction, de co-constitution d'une situation, les deux personnes côte-àcôte " métrisent » leur corporalité l'une par rapport à l'autre. Mais au-delà, portées potentiellement vers un même monde, vers un même projet, une même trajectoire, elles se rendent invisibles à la tierce personne arrivant sur elles. On pourrait croire à un manque de civilité et pourtant c'est juste un manque de co-mondanité. II y a en effet un conflit entre deux mises en situation, celle de la personne seule et celle du duo. L'absence de mise en situation partagée implique que le duo n'interprète pas la personne arrivant sur elle comme un acteur de la situation, il ne peut donc être opérateur spatial et donc modifier leur spatialité, le mouvement de leur corps sur la droite ou la gauche.

Une autre interprétation peut cependant s'ajouter et inscrire plus sociologiquement parlant les attitudes des trois protagonistes. En effet, que ce soit le duo ou le solo, chaque membre de la situation qui serait cette fois partagée du fait que chacun a bien conscience de la présence de l'autre/des autres, considère que cet autre/ces autres n'est pas/ne sont pas à leur place. Qu'en fonction de la désignation sociale qu'il/ils lui/leur donne(nt), il n'a pas/n'ont pas à être à cet endroitlà !

Une jeune fille court dans un bois public urbain. Elle suit normalement les sentiers très bien balisés et très utilisés par les habitants du coin. Elle a ses écouteurs sur les oreilles. Une autre personne la rejoint progressivement puis tout d'un coup la dépasse. Elle sursaute comme surprise de cette présence pourtant tout à fait normale et habituelle.

Si la présence du corps n'est pas attendue car pas entendue (que la personne ait ou non des appareils lui ôtant cette possibilité d'écoute du contexte), cette personne peut être concentrée, attentive à autre chose que le contexte. Que cela soit par l'intermédiaire d'un média technique ou non, diffusant ou non des musiques, les pensées peuvent être projetées sur un ailleurs qui constitue l'ici réellement vécu. La personne dépassée par le coureur plus rapide se retrouve alors dans un 
conflit de réalités, celle constituée par ses pensées et celle constituée par le contexte. Elle joue donc de diverses interspatialités, celle de la cospatialité avec le lieu où ses pensées l'amène et celle de la coprésence avec le contexte qui lui fait face. S'abstraire des sons du contexte environnemental, c'est potentiellement s'abstraire de sa relation corporelle à l'environnement. Sans dire évidemment que le corporel s'abstrait totalement des réalités coprésentes, celles-ci sont mises à distance pour laisser place à des éléments qui sont rapprochés à travers la musique produite. Henry Torgue rappelle ainsi que « beaucoup plus que la vue qui extériorise et distancie le décor, l'ouïe est vraiment le sens par lequel est ressentie l'immersion corporelle dans le milieu ambiant. Le sonore nous submerge, nous stresse ou nous apaise, réactivant toujours la conscience de notre échelle corporelle. C'est le corps producteur et récepteur de sons qui nous situe comme membres à part entière de l'environnement urbain : maîtres d'une situation par le contrôle acoustique ou, au contraire, exaspérés par des flux sonores subis. La sensation psychique de la proximité environnementale et de certaines de ses qualités passe par les relations sons-corps; $d$ 'une part, parce que nos oreilles n'ont pas de paupières pour interrompre l'écoute et, d'autre part, parce que le son, de nature ubiquitaire, se diffuse comme un enveloppement même si nous en localisons les sources. " (Torgue H., 2012, 48). La musique nous enveloppe et tend à supprimer certains éléments de notre perception qui sont habituellement des avertisseurs. Là, le sursaut est justement provoqué parce que l'événement n'est pas attendu, entendu.

Un homme d'une quarantaine d'années, apparemment seul dans son véhicule, circule en ville. Pourtant, il semble bouger les lèvres. Ses gestes et ses intentions du corps, traduites par des mimiques et des sourires, semblent évoquer une situation de communication avec une tierce personne, que suggère la présence d'un écouteur dans son oreille gauche. Il doit être au téléphone. Il arrive à un feu pour tourner à droite et devrait en théorie s'arrêter pour laisser passer des piétons. II n'obtempère pas pris dans sa conversation. L'accident est évité de justesse.

L'attention du conducteur est ailleurs car ses pensées sont attirées vers ce pour quoi ou pour qui il semble parler seul. Car sauf à être conçu comme fou, il doit être en relation avec un interlocuteur qui participe d'une situation commune qui fait fi du contexte objectivé. En effet, il ne parle pas avec un interlocuteur présent. Cette prise en compte du contexte objectivé aurait sans doute poussé le conducteur à réagir aux convenances du code de la route et à s'arrêter pour laisser passer le piéton. Il a en effet oublié les codes et les usages sociaux de la scène elle-même et surtout, il a oublié la présence de certains acteurs de celle-ci. Car sa mise en situation l'a invité à être ailleurs, pris dans une relation autre. Ce fait de ne pas s'arrêter pour laisser passer le piéton a d'ailleurs provoqué la mise en situation du contexte par d'autres observateurs qui ont pu alors s'offusquer de la scène. Et la trouver normale ou non, habituelle ou non.

On retrouve la même idée que précédemment car le conducteur et la joggeuse semblent penser être seuls dans le contexte qu'il et elle traversent, alors même qu'il y a d'autres participants de la scène, d'autres acteurs, qui potentiellement bougent, regardent ce qui se trame, et ont des stratégies d'arrangements spatiaux pour anticiper le déroulement de l'action. S'arrêter préventivement alors même qu'ils devraient passer, estimer la trajectoire que les autres acteurs vont emprunter et prévoir pour eux un autre cheminement qu'ils pensaient initialement pouvoir prendre.

Une différence tient en réalité au fait que si la joggeuse pratique son activité dans un espace public, on peut considérer qu'elle s'est mise en situation dans un espace clos, intime, du fait des écouteurs. Sa peur provoquée par le passage de ce coureur plus rapide indique qu'elle ne s'attend pas à cette présence. En revanche, le conducteur qui se trouve, dans une certaine mesure, dans un espace intime, la voiture, semble par son agitation dans celle-ci vouloir communiquer à son interlocuteur au téléphone mais finalement également à celles et ceux qui potentiellement le regardent au sein de l'espace public. En ce sens, la situation d'interaction qu'il opère avec son interlocuteur et qui structure ses intentions du corps renvoie à une mise en situation d'extimisation de ce qu'il est, de présentation de soi aux autres, qui ne participe pourtant apparemment pas de sa situation (mais bien seulement du contexte). Car si le fait de cette gestuelle convoque l'idée d'un parler haut et fort qui peut être lié à un problème de connexion, voire de surdité des deux protagonistes, il peut aussi être 
lié à la volonté d'extimiser certaines choses de la part du conducteur pour les autres acteurs supposés de la scène publique qu'il traverse, sans savoir s'il le fait ou non consciemment. C'est comme si cet habitant s'était abstrait de son environnement, tout en sachant qu'il est là mais aussi comme s'il avait oublié l'autre part des jeux d'interspatialités, celle de l'emboîtement des univers, des contextes. Son corps est certes dans un espace intime (la voiture) mais celui-ci n'est pas clos et il n'est donc pas invisible à l'espace public, à la mise en situation de cet espace pour un certain public.

Une famille est à table, un adulte discute avec un enfant qui lui raconte une histoire. Au même moment un autre enfant fait tomber son yaourt. L'adulte se détourne de la discussion n'écoutant plus ce que lui dit l'enfant pour se préoccuper du yaourt qui est en train de s'écouler lentement sur la table.

Les éléments d'attention peuvent être ou non attirés par des événements spatiaux qui se déroulent dans nos activités quotidiennes et perturber le cours "normal » des mises en situation. Peut mais n'implique pas forcément. En effet, l'adulte peut tout autant ne pas percevoir l'événement qui vient de se dérouler, soit que son in-attention le lui est rendue invisible pour lui soit qu'il se le soit rendu invisible volontairement à travers sa non réaction corporelle pour exprimer un sens aux deux enfants, celui qui parle et celui qui renverse le yaourt

Un enseignant épelle les noms de ses étudiants. A un certain moment, dans sa

liste, deux étudiants s'appellent tous deux Gaëtan. L'enseignant, au moment de dire le nom du premier Gaëtan se retourne sur le second. Le second lève le doigt puis se ravise.

Les intentions corporelles des acteurs peuvent devenir en situation des opérateurs spatiaux. Ici, le prénom épelé ne suffit pas à provoquer un mouvement du corps d'un des deux étudiants. En revanche, le regard porté vers l'un des deux induit en erreur ce dernier alors même qu'il n'est pas appelé. Ce mouvement corporel est donc indicatif d'une direction microsituationnelle.

Il est intéressant de terminer cet article par un dernier choc des situations paradoxal. Celui-ci se fonde sur l'absence objective de quoi que ce soit. Pourtant, ce rien est un opérateur spatial puissant. On doit à Emmanuelle Petit $(2009 ; 2012)$ d'en apporter les éléments de notre analyse.

Un guide de haute montagne emmène deux clients pour une course dans le massif du Mont-Blanc dans les Alpes du Nord. A plusieurs reprises, la voie qu'il emprunte se détourne du cheminement "classique" pour atteindre le sommet. Au bout d'un moment, intrigués par cette trajectoire inhabituelle, ses deux clients, amateurs chevronnés, l'interpellent. Le guide, embarrassé, finit par leur avouer qu'il a vu un de ses collègues mourir au fond d'une crevasse qui se trouve sur le cheminement classique et qu'il ne peut plus y passer.

Le corps absent peut être opérateur spatial car il est présent dans la situation que recompose de manière répétitive le guide. Pour s'échapper de ce souvenir permanent qui semble se réinviter à chacune des courses qui empruntent cette voie d'ascension, le guide préfère se détourner, mettre à distance pour pouvoir oublier. Car l'humanité, c'est aussi oublier, faire disparaître, faire ne plus exister, ne plus ek-sister, c'est-à-dire se permettre de ne plus devoir être auprès de. Pourtant, Patrick Baudry nous avertit : "Il est remarquable que le mot "disparition" puisse aujourd'hui remplacer le mot "mort". Pourtant pourrait-on dire d'une personne en train de mourir qu'elle est en train de disparaître ? Et les morts peuvent-ils être des disparus ? Ne faut-il pas une extravagante idiotie pour croire que les gens qu'on ne voit plus ne sont plus là ? " (Baudry P. 2004, pp.78-79). Car si l'habitant, en mourant, peut disparaître à son propre corps, et sans instruire la question de la réincarnation de notre esprit, il faut admettre qu'il ne peut disparaître complètement à l'esprit des autres qui pourtant ne voient plus ce corps. Ce corps mort, qu'il soit ou non encore visible demeure opérateur spatial pour celles et ceux qui demeurent à son écoute, à sa préoccupation, qui font mouvement corporellement pour aller à sa rencontre ou pour l'éviter ou corporéïquement pour s'y mettre à proximité mentalement. Le fait de vouloir, de devoir voir le corps mort part de ce principe de pouvoir l'assigner à un emplacement, son lieu d'inhumation, auprès duquel on se rendra par la mobilité du corps ou de l'esprit. Mais aussi pouvoir peut-être l'oublier. L'oubli étant souvent bien plus dur quand le corps mort n'est pas retrouvé comme c'est souvent le cas en Himalaya où ces derniers « ne sont pas rendus » par la montagne. 
L'ensemble de ces dispositifs situationnels types nous ont permis d'illustrer la nécessité de comprendre le corps, à travers sa mise en place, au sein d'une situation que constitue l'habitant. Cette constitution amène à repenser la géographie, en lui donnant une inflexion à la fois épistémologique (constitutivisme) et méthodologique (microgéographie). La première inflexion insiste sur le fait que chaque habitant configure l'espace de sa réalité et donc des spatialités du corps qui vont s'y opérer, s'y inscrire et donc y faire sens. Le corps placé l'est donc au sein d'une situation constituée par l'habitant (que celle-ci le soit co-constitutivement à travers un certain nombre de valeurs partagées, incorporées consciemment ou inconsciemment). Ce dernier ne fait donc pas avec un espace qui serait traduit objectivement par les différents acteurs supposés d'un contexte prédéterminé dans ses dimensions (le carrefour en tant qu'espace laboratoire) et les opérativités déterminantes que ce contexte pourrait engager (le conducteur doit s'arrêter au feu rouge du dit carrefour). Il fait avec un espace qu'il dimensionne en-deçà ou au-delà des éléments objectivement préhensibles par nos perceptions (au sens du réalisme naï) et au sein duquel il agit alors à travers ses mises en mouvements kinesthésiques, qu'ils soient musculaires ou à proprement parler spatiaux en tant qu'orientation des mouvements du corps. La seconde inflexion travaille les relations spatiales dans des micro-espaces d'interaction des corps, de leur mise en visibilité pour les autres, dans leur extimisation pratique (les gestes) et symbolique (l'habit, le maquillage, la coiffure). Elle invite à penser qu'à partir de micro-situations de la vie quotidienne, on peut comprendre quelque chose de bien plus complexe sur les spatialités et les socialités des habitants mais aussi que ces spatialités et socialités peuvent fonctionner structurellement de la même manière pour des espaces aux dimensions plus importantes.

\section{Bibliographie indicative}

Barbaras R., [1992]2008, " Motricité et phénoménalité chez le dernier Merleau-Ponty », in Richir M. et Tassin E. (ed.), Merleau-Ponty, phénoménologie et expériences, Grenoble, Jérôme Million.

Baudry P., (2004), Violences invisibles. Corps, monde urbain, singularité, Bègles, Éditions du Passant. Bourdieu P., ([1982]2001), Langage et pouvoir symbolique, Paris, Éditions Fayard.

Canetti E., ([1960]1966), Masse et puissance, Paris, Gallimard.

Céfaï D., (2007), " De la microphysique du pouvoir à l'ethnographie coopérative : itinéraires d'un pragmatiste ", in Joseph I., L'athlète moral et l'enquêteur modeste, Paris, Éditions Economica, coll. Études sociologiques, pp.1-47.

Coëffé V., (2014), "Le corps, un objet scientifique venu au monde ", L'Information Géographique, $\mathrm{n}^{\circ} 1$, Vol. 78, pp.6-26.

Di Méo G., (2009), « L'individu, le corps et la rue globale », Géographie et Cultures, n71, pp.9-23.

Duhamel A., (2014), "Corps, Espace, Monde. Enjeu(x) de l'objet de la géographie ", L'information géographique, $\mathrm{n}^{\circ} 1$, vol.78, pp.27-43.

EPEES, 2000, "Événement spatial », L’Espace géographique, n³, vol.29, pp.193-199.

Fournand A., (2005), " Le corps créateur d'espace: Le cas de la grossesse et de la naissance, in Fleuret Sébastien (dir.), Espaces, Qualité de vie et Bien-être, Angers, Presses de l'Université d'Angers, pp.297-305.

Giddens A., ([1984]1987), La constitution de la société : Eléments de la théorie de la structuration, Paris, PUF.

Goffman E., ([1963]1975), Stigmate. Les usages sociaux des handicaps, Paris, Les Éditions de Minuit. Houillon V., (1996), « Le libre jeu de l'espace et de l'imagination chez Heidegger : L'hésitation et le flottement de l'espace-temps ", Alter : Revue de phénoménologie, n4, pp.219-261.

Husserl E., ([1921]1974), Recherches Logiques. Tome 3: Eléments d'une élucidation phénoménologique de la connaissance (Recherche VI), Paris, PUF.

Jauréguiberry F., (2003), Les branchés du portable. Sociologie des usages, Paris, PUF.

Joseph I., (2003), Erving Goffman et la microsociologie, Paris, PUF.

Le Breton D., ([1992]2004), La sociologie du corps, Paris, PUF.

Lévy J., (1999), Le tournant géographique, Paris, Belin.

Lévy J. et Lussault M., (2003), Dictionnaire de la géographie et de l'espace des sociétés, Paris, Belin. 
Lussault M., (2007), L'homme spatial. La construction sociale de l'espace humain, Paris, Éditions du Seuil.

Lussault M., (2009), De la lutte des classes à la lutte des places, Paris, Éditions Grasset \& Fasquelle. Merleau-Ponty Maurice, (1945), Phénoménologie de la perception, Paris, Éditions Gallimard.

Merleau-Ponty Maurice, (1964), Le visible et l'invisible, Paris, Éditions Gallimard.

Merleau-Ponty Maurice, ([1964]1985), L'CEil et l'Esprit, Paris, Éditions Gallimard.

Mondada L., (2001), "L'entretien comme événement interactionnel », in Grosjean M. \& Thibaud J-P., L'espace urbain en méthodes, Marseille, Éditions Parenthèses, pp.197-214.

Petit E., (2009), "La lutte des places à Chamonix : Quand la mort devient enjeu spatial ", Cybergéo. Revue Européenne de Géographie, n475, URL : http://cybergeo.revues.org/22747

Petit E., (2012), Matérialisations du souvenir en montagne. Les enjeux identitaires des places et des placements, Bordeaux, Université Bordeaux 3, UMR 5185 ADESS, Di Méo G. (dir.), Thèse.

Pétonnet C., (1982), "L'observation flottante. L'exemple d'un cimetière parisien ", L'Homme, $n^{\circ} 4$, Tome 22, pp.37-47.

Quéré L., (1997), "La situation toujours négligée ? ", Réseaux, vol.15, n85, pp.163-192.

Revel J. (dir.), (1996), Jeux d'échelles. La microanalyse à l'expérience, Paris, Éditions de I'EHESS / Gallimard / Le Seuil.

Sloterdijk P., (2000), La domestication de l'être. Pour un éclaircissement de la clairière, Paris, Mille et une nuits (Arthème Fayard).

Tisseron S., (2001), L'intimité surexposée, Paris, Éditions Ramsay.

Tisseron S., (2011), "Intimité et extimité ", Communications, n88, pp.83-91.

Torgue H., (2012), " Cris et murmures de lieux », in Berque A., De Biase A. et Bonnin P. (dir.), Donner lieu au monde : La poétique de l'habiter, Paris, Éditions Donner Lieu, pp.39-51. 